\title{
Perception des messages nutritionnels par le consommateur français
}

\author{
Serge MICHELS \\ Protéines 11, rue Galvani, 75017 Paris \\ <smichels@proteines.fr>
}

\section{Contexte}

Le consommateur français est de plus en plus exposé aux messages nutritionnels par les différents acteurs de la société. Les adultes français n'ayant pas pour la plupart reçu de formation à la nutrition pour leur cursus scolaire, ils ont développé un savoir empirique quant à la nutrition. Ce savoir est nourri par l'abondante communication mise en œuvre par les principaux émetteurs que sont les pouvoirs publics, les mass media et les industriels.

À titre d'exemple, le nombre d'articles traitant de l'alimentation et de la santé dans les trente principaux supports de presse magazine français dépasse les 400 par an depuis au moins 1997 [1]. De même, le nombre de campagnes d'information des pouvoirs publics sur l'alimentation a fortement augmenté avec la mise en place du Programme national Nutrition Santé. Enfin, un nombre croissant de produits alimentaires porte un discours nutritionnel.

Face à cette surexposition du consommateur aux messages nutritionnels, peu d'études ont fait le point sur les connaissances du consommateur en matière de nutrition et leur perception de ces messages. Nous présentons ici une synthèse des principales études publiées en France et des enseignements que nous pouvons en tirer.

\section{Intérêt pour la nutrition}

Le lien entre alimentation et santé est aujourd'hui un lien très largement répandu dans les croyances du consommateur. Fischler et Rozine ont montré que ce lien perçu est commun à de nombreuses cultures, est probablement commun à l'espèce humaine et très ancien comme le montre la citation d'Hippocrate " que ton alimentation soit ta première médecine ».

La médecine moderne a largement contribué à conforter cette croyance puisqu'elle a démontré dans de nombreuses pathologies l'existence de liens entre habitudes alimentaires et apparition de ces pathologies.

De nombreux sondages ont été réalisés en France pour mesurer ce lien entre l'alimentation et la santé. Les résultats varient selon la formulation des questions posées, mais on observe dans tous les cas que l'on obtient au minimum des taux de $80 \%$ pour les personnes se déclarant d'accord avec une affirmation du type «ce que je mange a un impact sur ma santé ».

Au-delà de ce lien universellement connu du consommateur, plusieurs études ont cherché à mesurer plus précisément cet intérêt du consommateur pour la nutrition.

La plus récente [2], réalisée sur 850 consommateurs français, a montré que $63 \%$ des personnes interrogées se déclarent intéressées par la nutrition, alors que seulement $8 \%$ déclarent ne pas s'en préoccuper et $29 \%$ privilégier leurs seules habitudes alimentaires.

Ces chiffres semblent en augmentation si on les compare à l'étude de Poulain de 1998 [3], mais la formulation différente des questions ne permet pas d'en tirer des conclusions définitives.

De nombreuses études ont par ailleurs montré que cet intérêt pour la nutrition est en général plus affirmé chez les femmes que chez les hommes.

Par ailleurs, $85 \%$ des consommateurs [2] déclarent rechercher l'équilibre alimentaire. Les $15 \%$ qui ne déclarent pas rechercher l'équilibre alimentaire sont apparemment de jeunes adultes, des hommes, des personnes à faible niveau d'éducation et des personnes âgées.

\section{Source d'informations nutritionnelles}

Les sources $d$ 'information sur la nutrition déclarées sont par ordre d'importance : les médias (télévision, radio, presse écrite, magazines féminins...), les relations (famille, entourage, amis, collègues...), les fabricants (numéros verts et services consommateurs des fabricants, étiquettes et emballages des produits), les magazines ou livres spécialisés sur la santé ou la consommation et, enfin, les médecins, nutritionnistes, pharmaciens...

L'importance du discours nutritionnel des industriels apparaît clairement dans les études et notamment celui des allégations nutritionnelles en face avant des produits. En effet, la moitié des consommateurs français déclarent lire les allégations nutritionnelles dans le maga- sin alors que seul un quart des personnes déclarent lire le tableau des valeurs nutritionnelles.

\section{Connaissance des règles de l'équilibre alimentaire}

Le manque de connaissance en nutrition est I'une des raisons fréquemment invoquées pour justifier la non-observance des règles de la diététique moderne. II est donc intéressant de mesurer le degré de connaissance des individus de normes diététiques.

L'étude INCA [4] permet de répondre en partie à cette question. Elle présente notamment l'intérêt de fournir des résultats triés en fonction de l'origine sociale des individus et sur un échantillon représentatif de la population française.

Elle montre que dans les principaux messages émis par les pouvoirs publics, à savoir la limitation de la consommation de sucre et de matières grasses, ont été assimilés par les consommateurs. Elle conforte les résultats de nombreux focus groups que nous avons pu réaliser par ailleurs et qui ont mis en évidence une bonne connaissance des principales règles de l'équilibre alimentaire.

Ceci pose donc la question des messages de santé publique sur ces nutriments. Les messages doivent-ils viser à communiquer à l'ensemble de la population la norme nutritionnelle et encourager ainsi de meilleures pratiques diététiques, ou cibler les messages afin de toucher les consommateurs dont les pratiques sont les plus éloignées de la norme?

Le tableau 1 montre que l'on observe de faibles différences de connaissances de la norme selon le niveau social, alors que l'on sait par ailleurs qu'il existe des différences de pratiques très importantes selon le niveau social.

\section{Connaissance du rôle des nutriments}

L'étude DGAL/CLCV, à laquelle nous avons participé, a permis d'apporter quelques éclairages nouveaux sur la connaissance réelle des nutriments

La plupart des études s'attachent uniquement à demander à la personne interviewée si elle 
Tableau 1. Éléments nutritionnels à éviter selon la catégorie socio-professionnelle. INCA Crédoc. 2000.

\begin{tabular}{|lccc|}
\hline $\begin{array}{l}\text { Eléments nutritionnels } \\
\text { à éviter }\end{array}$ & Cadres & Ouvriers & Employés \\
\hline Matières grasses & 61,3 & 55,4 & 59,8 \\
Sucre & 41,2 & 28,9 & 34,3 \\
Cholestérol & 28,1 & 27,4 & 30,5 \\
Aucun & 19,1 & 21,7 & 17,8 \\
Acides gras saturés & 15,1 & 11,7 & 13,1 \\
Sel & 7,0 & 14,4 & 12,7 \\
Acides gras polyinsaturés & 4,5 & 3,0 & 2,5 \\
\hline
\end{tabular}

connaît un nutriment. Elles ne permettent donc pas de mesurer la véritable connaissance puisqu'elles s'appuient sur du déclaratif. L'étude DGAL/CLCV a proposé à 850 interviewés plusieurs propositions de définition, justes et fausses, pour quelques nutriments afin de quantifier les taux de bonnes comme de mauvaises réponses.

Les résultats montrent que :

- $23 \%$ des Français ne savent pas ce que sont les glucides, ou les confondent avec d'autres ingrédients ou nutriments.

- $39 \%$ des Français ne savent pas si les acides gras saturés sont bons ou mauvais pour leur santé, et $19 \%$ pensent que ces derniers sont bons pour la santé, soit au total $58 \%$ des personnes interrogées qui ne peuvent utiliser une telle information.

Ces résultats confirment que globalement :

- Le consommateur connaît les règles de la diététique lorsque l'on raisonne en aliments.

- Un nombre important de consommateurs ignore le rôle exact des nutriments.

- On peut raisonnablement penser que la grande majorité des consommateurs n'ont aucun repère en matière de quantités de nutriments à consommer quotidiennement.

Ce type d'information montre clairement les limites de l'étiquetage nutritionnel en particulier dans sa forme réglementaire dans I'Union européenne. S'il est tout à fait légitime de considérer que le consommateur doit être informé sur le contenu nutritionnel des aliments mis à disposition, il faut relativiser la portée de ces informations en terme d'éducation du consommateur dans la mesure où les trois quarts d'entre eux déclarent ne jamais lire l'étiquetage nutritionnel que ce soit sur le point de vente ou au domicile.

De plus, les études montrent que les allégations nutritionnelles sont considérées comme beaucoup plus simples à comprendre que le tableau des valeurs nutritionnelles (TVN), ce qui semble tout à fait logique. En effet, le TVN est une information brute chiffrée sans clés d'interprétation alors que l'allégation met en avant un bénéfice pour le consommateur de façon explicite.

\section{Perception des messages nutritionnels}

L'étude de la perception des messages nutritionnels par les consommateurs fait appel à des études qualitatives notamment des focus groups.

Nous avons eu l'occasion de réaliser de nombreux groupes de consommateurs (données non publiées) en France et dans d'autres pays européens qui, associés aux travaux des sociologues notamment Fischler et Poulain, permettent de comprendre certains déterminants de la perception des messages nutritionnels.

Pour comprendre la perception d'un message nutritionnel, il faut analyser les interactions entre différents éléments :

- Le support : il s'agit de l'aliment auquel on prête des vertus nutritionnelles.

- Le bénéfice : il s'agit de la promesse faite au consommateur.

- La justification : il s'agit du support rationnel (par exemple : du calcium pour les os) ou holistique (par exemple : « source de jeunesse ») de la promesse.

Notre expérience montre que l'élément-clé en termes de perception n'est pas la justification mais l'interaction entre le bénéfice et le support. En effet, même s'il est évident que la promesse nutritionnelle doit être supportée par une réalité objectivable, les aliments ne sont pas des aliments vierges de toute représentation et l'on ne peut lui attribuer toutes sortes de vertus.

Nos aliments sont inscrits dans une culture, des rites sociaux et culinaires et l'on ne peut leur appliquer les recettes marketing de la lessive poudre blanche neutre et dénuée de représentations symboliques.

Les travaux des sociologues et notamment le principe d'incorporation [5], nous apportent des clés pour comprendre les perceptions des consommateurs. Imaginons par exemple un industriel souhaitant mettre sur le marché de la viande de lion en France, et lui accorder un bénéfice santé, que l'on supposera supporté sur le plan scientifique, de calme et de bien-être pour le dîner avant d'aller se coucher. Cette promesse entrera en dissonance avec nos croyances selon lesquelles «manger du lion» fournit de l'énergie voire de l'agressivité. Ainsi on comprend aisément qu'un aliment animal ne peut porter les mêmes promesses de la même façon qu'un aliment végétal.

Nous touchons ici un élément-clé de ce que I'on appelle la « cacophonie nutritionnelle ». Les industriels financent de nombreuses recherches sur les vertus nutritionnelles de leurs produits. Mais les bénéfices nutritionnels, parfois parfaitement justifiés sur le plan scientifique, peuvent être en complète opposition avec nos représentations et nos symboles.

\section{Conclusion}

L'industriel devra donc manipuler avec précaution les allégations afin de ne pas participer à la perte de repères du consommateur, et ce, alors que le consommateur est aujourd'hui exposé à une abondance croissante de messages nutritionnels.

En particulier on observe parfois la volonté, pour des raisons de concurrence, de mettre en avant des bénéfices différents sur un même produit ou d'utiliser des justifications différentes (omégas 3, 69 par exemple) pour un même bénéfice.

Le risque, même si des messages peuvent présenter un intérêt économique à court terme, est d'hypothéquer le long terme et de susciter un rejet en masse des messages nutritionnels par le consommateur. Si aujourd'hui les messages nutritionnels restent un moyen efficace de donner de la valeur aux marques et produits, des signes suffisamment forts montrent que la forte pression publicitaire sur certains messages et l'excès de signaux contradictoire sur les produits peuvent présenter un risque à moyen terme pour les industriels.

\section{RÉFÉRENCES}

1. MICHELS S. Etude Nutrimedia. 2000; (Non Publié).

2. CLCV-DGAL. Étude de la compréhension par les consommateurs de certaines mentions figurant dans l'étiquetage des denrées alimentaires préemballées et à leur perception de certaines allégations nutritionnelles, fonctionnelles et de santé. 2004.

3. POULAIN JP. Les jeunes seniors et leur alimentation. Cahiers de l'OCHA 1998 : 9 ; (Paris).

4. INCA CREDOC. 2000.

5. FISCHLER C. L'homnivore. Paris : Odile Jacob, 2000. 